\title{
Effects of interactive video-game-based exercise on balance in older adults with mild-to-moderate Parkinson's disease
}

Rey-Yue Yuan ${ }^{1,2}$, Shih-Ching Chen ${ }^{3,4,5}$, Chih-Wei Peng ${ }^{5,6,7}$, Yen-Nung Lin ${ }^{8,9}$, Yu-Tai Chang ${ }^{4,5}$ and Chien-Hung Lai ${ }^{3,4,5^{*}}$

\begin{abstract}
Background: This study aimed to evaluate the effectiveness of a customized interactive video game-based (IVGB) training on balance in older adults with mild-to-moderate Parkinson's disease (PD).

Methods: In this 12-week crossover trial, PD patients $\geq 65$ years of age were randomly divided into Group A (a 6week intervention phase followed by a 6-week control phase) and Group B (a 6-week control phase followed by a 6-week intervention phase). Participants received IVGB exercise training during the intervention phase and no exercise during the control phase. Functional outcomes were measured using behavioral evaluation scales and questionnaires at baseline, week 6 and week 12.

Results: Twenty-four PD patients were included in this study, and were evenly divided into two groups. After Bonferroni adjustment, the changes in Modified Falls Efficacy Scale (MFES) and two subscales of Multi-Directional Reach Test were significantly different between two groups in the first 6-week period. In addition, the changes in Berg Balance Scale, MFES, and two subscales of Maximum Step Length were significantly different between two groups in the second 6-week period. Compared to controls, 6-week IVGB exercise intervention significantly improved different but overlapping functional outcomes in two groups of PD patients.
\end{abstract}

Conclusions: The customized IVGB exercise training improves balance, postural stability and confidence in preventing falls in older adults with mild-to-moderate PD. However, this IVGB exercise doesn't have a significant impact on quality of life.

Trial registration: ClinicalTrials.gov. NCT03689764. Registered 27 September 2018, retrospectively registered.

Keywords: Interactive video game-based exercise, Parkinson's disease, Balance, Crossover trial

\section{Background}

Parkinson's disease (PD) is the second most common neurodegenerative disease affecting older adults [1]. Neurological deficits in PD affect the musculoskeletal and balance systems, thereby impairing mobility, postural

\footnotetext{
* Correspondence: chlai@tmu.edu.tw

${ }^{3}$ Department of Physical Medicine and Rehabilitation, School of Medicine, College of Medicine, Taipei Medical University, Taipei, Taiwan

${ }^{4}$ Department of Physical Medicine and Rehabilitation, Taipei Medical University Hospital, No. 252, Wu-Hsing St., Taipei City 110, Taiwan Full list of author information is available at the end of the article
}

stability, and walking capacity [2, 3]. The clinical symptoms and psychosocial effects of PD often limit the participation of PD patients in social and physical activities, which subsequently further declines in functional status [4]. Restricted mobility in PD patients contributes to secondary health complications and higher treatment costs [5]. Exercise programs benefit PD patients, improving physical strength and function, health-related quality of life, balance, and gait speed to some degree [6, 7]. A cohort study with one-year follow-up revealed that higher frequency of physical activity is associated with a reduced

(c) The Author(s). 2020 Open Access This article is licensed under a Creative Commons Attribution 4.0 International License, which permits use, sharing, adaptation, distribution and reproduction in any medium or format, as long as you give appropriate credit to the original author(s) and the source, provide a link to the Creative Commons licence, and indicate if changes were made. The images or other third party material in this article are included in the article's Creative Commons licence, unless indicated otherwise in a credit line to the material. If material is not included in the article's Creative Commons licence and your intended use is not permitted by statutory regulation or exceeds the permitted use, you will need to obtain permission directly from the copyright holder. To view a copy of this licence, visit http://creativecommons.org/licenses/by/4.0/. The Creative Commons Public Domain Dedication waiver (http://creativecommons.org/publicdomain/zero/1.0/) applies to the data made available in this article, unless otherwise stated in a credit line to the data. 
risk of cognitive and motor decline in old adults with mild parkinsonian signs [8]. However, most older people seldom participate in exercise programs because the content of conventional programs may be repetitive, uninviting, or difficult [9]. Hence, it is clinically important to develop an exercise program that is easy and interesting to older adults with mild-to-moderate $\mathrm{PD}$, thereby encouraging patient participation and subsequently ameliorating PDrelated motor symptoms.

Auditory and visual biofeedback effectively improves postural stability and balance in healthy adults [10]. Visual cues, combined with treadmill training, result in greater improvements in gait in patients with Hoehn and Yahr (HY) stage 2-4 PD than treadmill training alone [11]. A study of older patients with HY stage 2-3 PD suggested that either auditory or visual cues increase exercise intensity in a virtual cycling system [12]; however, the superiority of auditory over visual cues in ameliorating PD motor symptoms was indicated by a meta-analysis [13]. In addition, a systematic review concluded that gamifying visual feedback and providing performance feedback in real-time facilitate movement rehabilitation in PD [14].

The safety and effectiveness of several commercial exercise gaming (exergaming) systems for motor rehabilitation in PD patients have been extensively investigated [15-21]. Two prospective studies reported that exercises using the virtual-reality-based Wii Fit substantially improved obstacle-crossing performance, dynamic balance, and attention in patients with mild-to-moderate PD (HY stage 3 or lower) [19, 21]. Similarly, two randomized controlled trials demonstrated that PD patients with HY stage 3 or less benefit from commercial exergames during physical rehabilitation $[17,20]$. A recent short review found that most of the commercially available rhythmbased games were insufficient for training rhythmic skills, although some features of the games were interesting. Hence, this review concluded that current rhythm-based games still required further modification to devise efficient rhythmic training programs for patients with motor or cognitive impairment [15]. A systematic review published in 2014 concluded that commercial exergames (Nintendo Wii Fit platform and Sony Playstation Eye) often require fast and complex responses that may be too difficult for some PD patients; therefore, specifically tailored exergames are needed to meet the safety and rehabilitation needs of patients with mild-to-moderate PD [16]. A systematic review of PD rehabilitation using commercial exergames in 2014-2017 concluded that exergame-based PD rehabilitation is equal to or more effective than traditional PD rehabilitation [18]. Nevertheless, additional randomized controlled trials (RCTs) that assess more standard outcomes, such as Universal Parkinson's Disease Rating Scale (UPDRS), Berg Balance Scale (BBS), and
Timed Up and Go (TUG) tests, are warranted to confirm the effectiveness of exergames in PD rehabilitation [18].

To meet the unique needs of specific user groups, several customized interactive video-game-based (IVGB) exercise systems have been developed [22-24]. Compared to repetitive conventional physical activities, IVGB training provides an interesting and interactive environment, so participants would be more likely to enjoy completing their physical activity regimen. IVGB exercise is reported to improve dynamic balance control and attention span in patients with traumatic brain injury [22]. Our previous crossover study found that IVGB exercise enabled healthy adults aged $\geq 65$ years to significantly improve scores on the Berg Balance Scale, Modified Falls Efficacy Scale (MFES), and Unipedal Stance Test (UST) and shorten TUG test completion time [24]. In another crossover study, we found that IVGB intervention enhanced BBS scores and reduces TUG task completion time significantly in diabetic patients with peripheral neuropathy [23]. Herein, we hypothesized that IVGB training may improve balance in older adults with mild-to-moderate PD. To examine this hypothesis, the present randomized crossover 12-week trial was conducted in community-living ambulatory elderly patients with mild-to-moderate PD.

\section{Methods \\ Participants}

The study cohort included PD outpatients who were recruited from the Neurology Department of Taipei Medical University Hospital, Taiwan. The inclusion criteria were: i) aged 60 to 80 years; ii) clinical diagnosis of idiopathic mild-to-moderate PD of HY stage 1-3 [25]; iii) independent community-living ambulatory individuals; and iv) cognitive level as assessed by the Mini-Mental Status Examination (MMSE) score $>23$. The exclusion criteria were: i) history of dementia, previous stroke, arthritis, vision impairment, diabetes, or uremia; ii) previous engagement in any exergaming training program or commercial exergaming system within 6 months; and iii) inability to walk without assistance or the presence of cardiovascular disease that impaired walking.

\section{Ethical considerations}

The study protocol was reviewed and approved by the Joint Institutional Review Board of Taipei Medical University (approval number: TMU-JIRB 201311032). The potential risks and benefits were explained to each participant in advance, and all participants provided signed informed consent before taking part in the current study. The study is registered at ClinicalTrials. gov (identifier number: NCT03689764). 


\section{Study design}

In the present prospective, randomized, single-blind, crossover 12-week trial, community-living ambulatory older adults with mild-to-moderate PD were equally divided and randomly assigned to either Group A or Group B. The participants in Group A received IVGB training during the first 6 weeks (intervention phase), followed by no IVGB exercise during the subsequent 6 weeks (control phase); the participants in Group B had no IVGB exercise intervention during the first 6 weeks (control phase) and then received IVGB training in the following 6 weeks (intervention phase) (Fig. 1). Outcomes measured at the end of 6 and 12 weeks were compared between the groups. The crossover design decreased the variability between groups, because the same patients served as the controls and experimental subjects during different time periods as previously described [26].

\section{IVGB intervention}

The IVGB system was developed by modifying the XaviX entertainment system (SSD Company Limited, Shiga, Japan). We previously reported the beneficial effects of the IVGB system on motor recovery [23, 24]. The XaviX system was originally designed for healthy users. Hence, we modified the difficulty levels and scoring system of the XaviX system for older adults with mild-to-moderate PD, and the step mat was fixed on the floor to minimize the risk of falls. The IVGB exercise program consisted of two tasks: a multi-directional step task and a target-directed stepping task. The IVGB system offers aural and visual feedback in both tasks to increase participants' attention. First, the participant followed the illustrated instructions shown on the monitor to step on the target area to complete the multidirectional step task (Fig. 2). This first task assesses the participant's capability for weight-shifting, dynamic balance, and stability. The participant then followed the illustrated instruction to complete the target-directed stepping task (Fig. 3). This second task evaluates the participant's movement coordination and balance while standing on one leg. Adjustments were made between three levels of difficulty and direction of steps by a physical therapist in accordance with the participant's cognition, attention, balance, walking ability, strength, and weight-shifting ability. To ensure uniformity in exercise posture, participants were asked to maintain an upright position and to avoid compensating by postural sway. All IVGB training sessions were held at Taipei Medical University Hospital and were instructed and monitored by a certified physical therapist who was responsible for ensuring participant safety during the exercises.

Exercise performance measurements, including time to complete, number of successful steps, and avatarmimicking foot trajectory, were displayed on the monitor in real time during IVGB training and summarized at the end of the training. During the 6-week training (intervention phase), participants reported to the hospital 3 days each week to complete the 30-min training (15 min per task).

\section{Outcome measures}

Outcome measures were assessed at the end of the first 6week intervention/control phase and the end of the subsequent 6-week intervention/control phase to determine the effects of IVGB training (Fig. 1). The primary outcome measure was the BBS score; the secondary outcome measures included scores on the 36-Item Short-Form Health Survey (SF-36), MFES, Multi-Directional Reach Test (MDRT), and Maximum Step Length (MSL) test.

\section{BBS}

The BBS objectively assesses balance ability via 14 functional balance tasks, and has been confirmed effective in PD patients [27]. The BBS applies a 4-point scale, in which a score of 0 indicates inability to perform the task and a score of 4 indicates task completion. A total BBS score less than 46 points indicates the risk of falls [28].

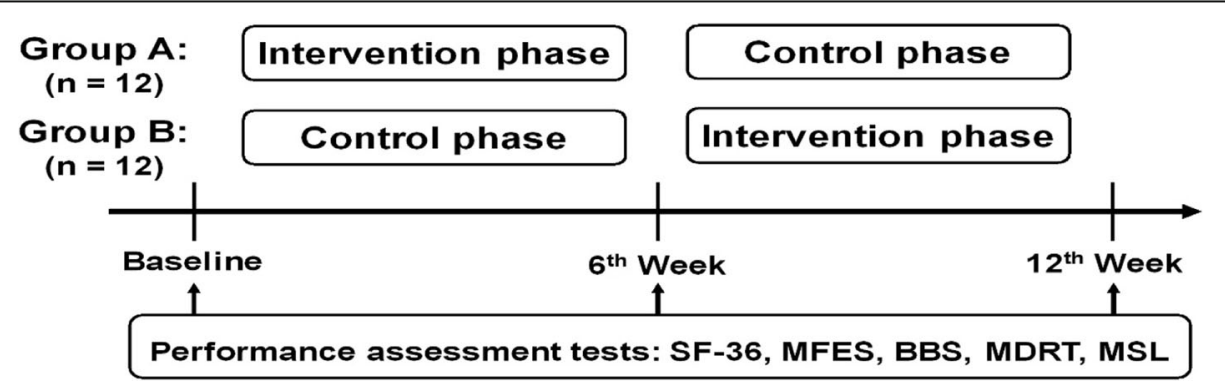

Fig. 1 Experimental design. Twenty-four participants with mild-to-moderate PD were randomly assigned to Groups A and B. Participant performance was assessed before intervention (baseline) and at weeks 6 and 12. Group A participated in the interactive video-game-based (IVGB) exercise program during the first 6 weeks, followed by a 6 -week period without exercise. Group B participants did the reverse, starting with 6 weeks without exercise followed by 6 weeks of the IVGB exercise program. SF-36, 36-Item Short-Form Health Survey; MFES, Modified Falls Efficacy Scale; BBS, Berg Balance Scale; MDRT, Multi-Directional Reach Test; MSL, Maximum Step Length 

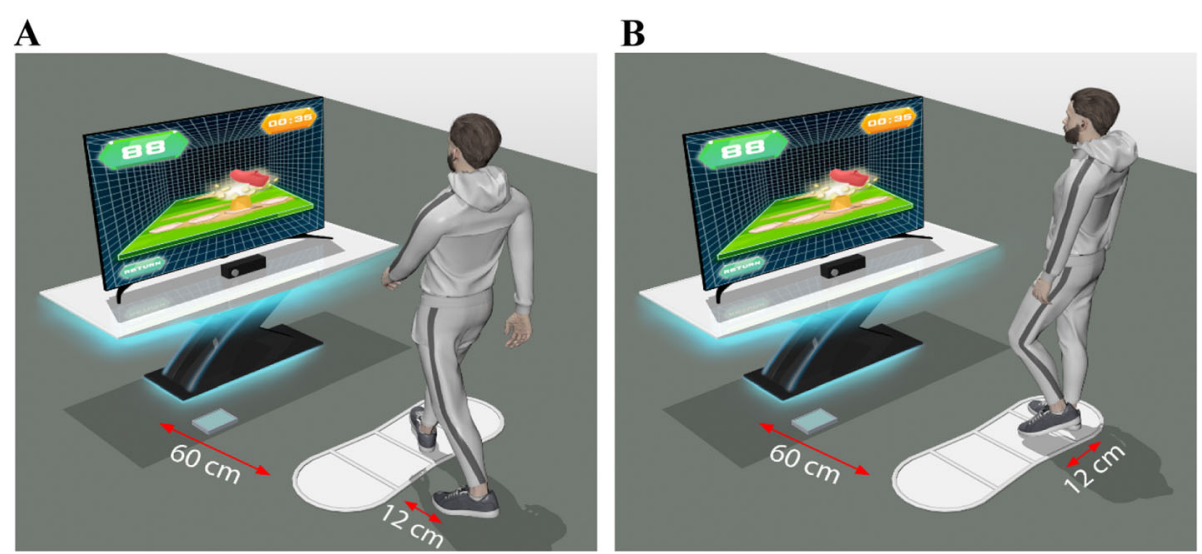

C

D
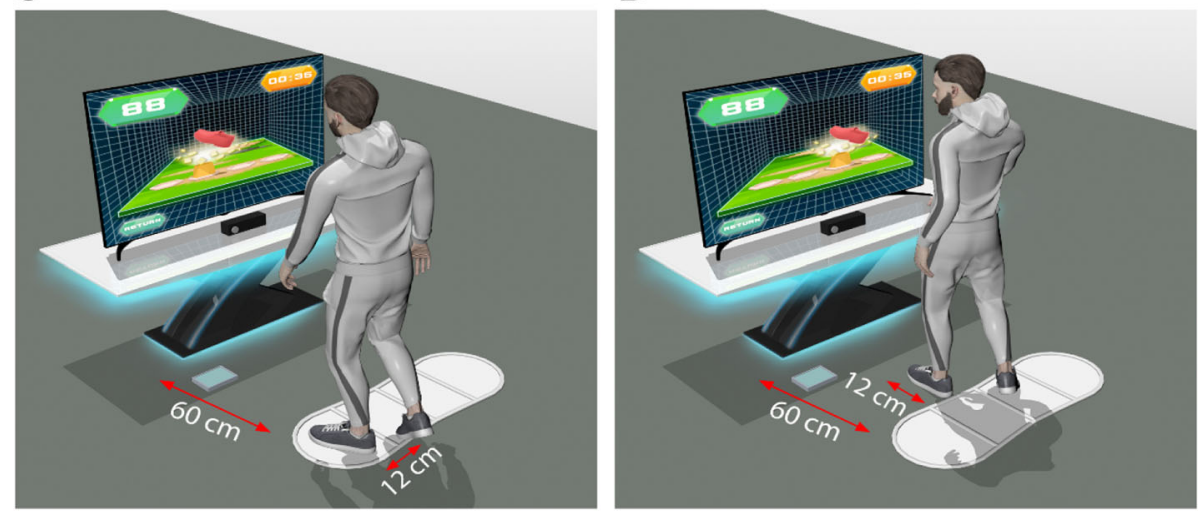

$\mathbf{E}$

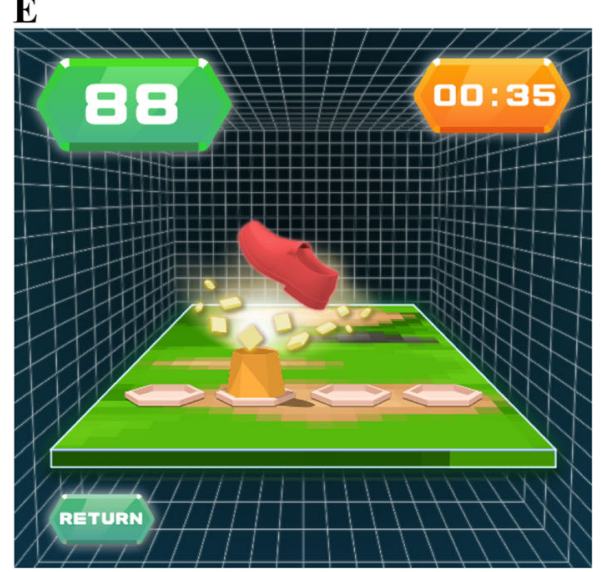

Fig. 2 Schematic diagram of the multidirectional reaching task. The stepping mat was placed $60 \mathrm{~cm}$ in front of the monitor. a: Participants performed interactive multidirectional reaching tasks by following the target appearing on the television screen, with movements tracked by infrared photosensors in the controllers. $\mathbf{b}$ \& $\mathbf{c}$ : For the antero-posterior stepping practice, participants stood $12 \mathrm{~cm}$ away from the mat in front/ behind and stepped onto the target area according to the monitor cues. $\mathbf{d} \& \mathbf{e}$ : For the medial-lateral stepping practice, participants stood on the right/left side of mat (starting area) and stepped onto the target area according to the monitor cues. For forward-backward and medial-lateral stepping, the floor and mat were marked to ensure that participants started and ended in the same place

\section{SF-36}

The SF-36 is a self-reported quality of life questionnaire that assesses health status of 8 domains, including physical functioning (10 items), social functioning (2 items), physical problems (4 items), emotional problems (3 items), general mental health (5 items), vitality (4 items), bodily pain ( 2 items), and general health (5 items). Each item is scored from 0 (worst Health) to 100 (best health) [29]. The SF-36 has been wildly used to evaluate the impact of PD on quality of life [30, 31]. 

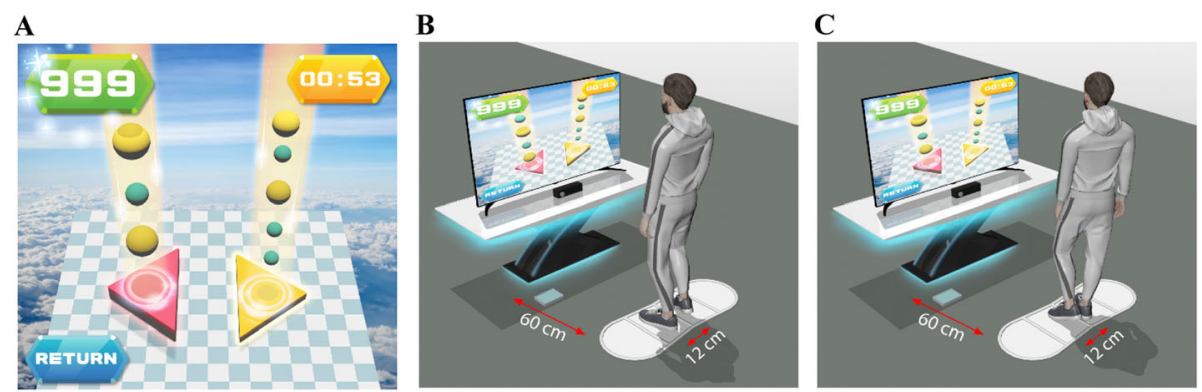

Fig. 3 Schematic diagram of the target-oriented stepping task. The stepping mat was placed $60 \mathrm{~cm}$ in front of the monitor. Participants stood on the marked area of the mat as the starting point. a: Participants performed the interactive target-oriented stepping task according to the cue on the television screen, with motion monitored by infrared photosensors in the controllers. b: Right foot was raised according to the cue on the monitor. c: Left foot was raised according to the cue on the monitor

\section{MFES}

The MFES is a 14-item questionnaire regarding daily indoor and outdoor physical activities and is a valid tool for measuring fall-related self-efficacy in PD [32].

\section{MDRT}

The MDRT is a valid assessment of stability in multiple directions [33]. The participants were required to reach in the forward, left, and right directions. For the forward-reaching direction, the participant raised both arms to the shoulder level, reached forward as far as possible without moving the feet, and then maintained the end-range position for $3 \mathrm{~s}$. The distance reached by the middle finger was measured. Similar methods were used to assess right and left lateral reach. The assessment was performed 3 times in each direction, and the data were averaged for further statistical analysis.

\section{MSL}

The MSL test is a valid measurement of stepping ability and serves as an indicator of mobility function and fall risk in older adults [34]. Participants performed the MSL test in the forward, lateral, and backward directions. For the forward direction test, the participant crossed the arms over the chest, took a maximum step forward with one leg only, and then returned to the starting position with a single step. The stepping distance was measured. The same method was used the lateral and backward direction tests.

\section{Statistical analysis}

Continuous variables are presented as the mean \pm standard deviation (SD), and the corresponding betweengroup differences were analyzed using Student's t-test. Categorical variables are presented as the count and percentage, and the corresponding between-group differences were analyzed using the chi-square test or Fisher's exact test. Two-tailed $P<0.05$ indicated statistical significance for the baseline characteristic analysis. To test for differences in outcome measures between time points (baseline, week 6 and week 12), repeatedmeasures ANOVA was performed separately for two different patient groups. To examine difference in changes in outcome measures between Group A and Group B, ttest was performed separately for two distinct 6-week periods. Since SF-36, MDRT and MSL contain multiple subscales, $P$ values of the subscales of SF-36, MDRT and MSL were corrected using the Bonferroni method. The alpha value was set to $P=0.05$ / (number of subscales $\mathrm{x}$ number of categories); categories could be time points or patient groups in the present study. Hence, the Bonferroni correction was conducted in between-time point comparison of each subscale in the same group, and in between-group comparison of the change in each subscale in the same 6-week period. All statistical analyses were performed using SAS version 9.4 (Windows NT version, SAS Institute, Inc., Cary, NC, USA).

\section{Results}

A total of 24 community-living older adults with mildto-moderate PD were included in this prospective crossover study and were randomly divided into two groups: Group A and Group B (Fig. 1). The demographic and clinical characteristics of two groups of PD patients are shown in Table 1. Age, body height, body weight, body mass index (BMI), leg length, foot length, fall in the past year, and HY stage were comparable between two groups. However, the MMSE score and percentage of females were significantly lower in Group B than in Group A (Table 1).

The outcome measures of the two groups at various time points are shown in Supplemental Table 1. After the Bonferroni correction, no significant differences in all outcome measures between time points were found in Group A. In contrast, in Group B, BBS at week 12 was significantly higher than those at baseline and at week 6 (Fig. 4a). In addition, three subscales of MSL, including the right side ( $\mathrm{R}$. Side), the posterior side of the 
Table 1 Baseline demographic and clinical characteristic of two groups of PD patients

\begin{tabular}{|c|c|c|c|}
\hline \multirow{4}{*}{. } & Group A & Group B & \multirow[t]{2}{*}{$P$-value } \\
\hline & $N=12$ & \multirow{2}{*}{\multicolumn{2}{|c|}{$N=12$}} \\
\hline & Mean \pm SD & & \\
\hline & $67.8 \pm 5.5$ & $66.5 \pm 8.8$ & $0.66^{\mathrm{a}}$ \\
\hline Body height $(\mathrm{cm})$ & $158.5 \pm 7.4$ & $164.3 \pm 7.5$ & $0.07^{\mathrm{a}}$ \\
\hline Body weight $(\mathrm{kg})$ & $58.0 \pm 10.9$ & $61.1 \pm 6.3$ & $0.42^{\mathrm{a}}$ \\
\hline $\mathrm{BMI}\left(\mathrm{kg} / \mathrm{m}^{2}\right)$ & $23.2 \pm 4.6$ & $22.7 \pm 2.4$ & $0.75^{\mathrm{a}}$ \\
\hline \multicolumn{4}{|l|}{ Leg length (cm) } \\
\hline Left leg & $79.5 \pm 4.5$ & $81.1 \pm 5.2$ & $0.44^{\mathrm{a}}$ \\
\hline Right leg & $79.6 \pm 4.8$ & $81.2 \pm 5.6$ & $0.46^{\mathrm{a}}$ \\
\hline \multicolumn{4}{|l|}{ Foot length $(\mathrm{cm})$} \\
\hline Left foot & $23.3 \pm 1.1$ & $24.2 \pm 1.6$ & $0.10^{\mathrm{a}}$ \\
\hline Right foot & $23.3 \pm 1.1$ & $24.2 \pm 1.6$ & $0.11^{\mathrm{a}}$ \\
\hline \multirow[t]{2}{*}{ MMSE } & $28.5 \pm 1.7$ & $26.0 \pm 2.6$ & $0.01^{a}$ \\
\hline & N (\%) & & \\
\hline \multicolumn{4}{|l|}{ Sex } \\
\hline Female & $10(83.33 \%)$ & $3(25 \%)$ & $0.004^{b}$ \\
\hline Male & $2(16.67 \%)$ & $9(75 \%)$ & \\
\hline \multicolumn{4}{|c|}{ Fall in the past year } \\
\hline No & $12(100 \%)$ & $8(66.67 \%)$ & $0.09^{c}$ \\
\hline Yes & $0(0 \%)$ & $4(33.33 \%)$ & \\
\hline \multicolumn{4}{|c|}{ Hoehn and Yahr staging scale } \\
\hline Stage 1 & $4(33.33 \%)$ & $3(25 \%)$ & $0.77^{c}$ \\
\hline Stage 2 & $5(41.67 \%)$ & $4(33.33 \%)$ & \\
\hline Stage 3 & $3(25 \%)$ & $5(41.67 \%)$ & \\
\hline
\end{tabular}

$S D$ standard deviation, $N$ number, $\%$ percentages, $B M I$ Body mass index, MMSE Mini-mental state examination

${ }^{\mathrm{a}}$ T Test; ${ }^{\mathrm{b}}$ Chi-square Test; ${ }^{\mathrm{c}}$ Fisher's Exact Test

Significant difference $(p<0.05)$ was indicated in bold

right leg (R. Post) and the posterior side of the left leg (L. Post), were significantly higher at week 12 than at baseline (Fig. 4b-d).

In addition, between-group comparison of the changes in outcome measures in the first 6-week period (values at week 6 minus values at baseline) and in the second 6week period (values at week 12 minus values at week 6) were conducted separately (Table 2). After the Bonferroni correction, the changes in MFES and two subscales of MDRT, including MDRT to the right side (MDRT-R) and MDRT-L, were significantly different between two groups in the first 6-week period. Moreover, the changes in BBS, MFES, and two subscales of MSL (R. Post and L. Post) were significantly different between two groups in the second 6-week period (Table 2).

In contrast to BBS, MFES, MDRT and MSL, IVGB training did not significantly altered any subscale of SF36 (Table 2). In addition, no adverse effects were observed during the entire study period.

\section{Discussion}

In this prospective crossover study, we found that a 6week IVGB exercise program significantly enhanced confidence in preventing falls, as evidenced by higher scores on MFES, in all included older outpatients with mild-to-moderate PD. In addition, 6-week IVGB training significantly improved the ability of balance and the capability of stepping their leg in the left, right and back directions in patients undergoing IVGB training in the second period. However, no significant impact of IVGB training on quality of life was found in this study. Thus, IVGB intervention significantly improved different but overlapping functional outcomes in two groups of older patients with mild-to-moderate PD.

In the present study, IVGB training significantly improved BBS in the second 6-week period, but not in the first 6-week period, which might be in part due to the fact that the mean baseline BBS score of the PD patients undergoing IVGB training in the second period was lower than that of those undergoing IVGB training in the first period ( $36.2 \pm 8.9$ vs. $50.7 \pm 3.5)$. Supporting our speculation, a recent study reported that PD patients with lower baseline BBS scores achieve greater improvements in balance rehabilitation that those with higher BBS scores do [35]. In addition, improvements in balance using conventional balance training are suggested to be influenced by cognitive reserve, an individualspecific active expression of the brain's ability to respond to physical damage [35]. In the present study, compared to PD patients undergoing IVGB training first, PD patients undergoing IVGB training in the second period had more male patients but a lower mean MMSE score, which might also partially contribute to the different results between groups. Hence, additional studies with a larger sample size are warranted to investigate the extent to which sex and mental status affect the effectiveness of IVGB training.

Depression and anxiety are commonly noted in PD patients, and both are highly associated with worsening motor function, symptom fluctuations, cognitive losses, and poor quality of life [36]. Game-based exercise training provides not only physically challenging exercise but also cognitive stimulation, thereby boosting both physical and mental performance [37]. A systematic review concluded that exergaming provided an innovative, fun, and relatively safe method of exercise and had significant effects on physical and cognitive function in persons with dementia [38]. The present study demonstrated that 6 -week IVGB training significantly elevated MFES scores in all included PD patients, indicating that PD patients' confidence in avoiding falls was boosted after IVGB training. Such beneficial effect of IVGB training on confidence in avoiding falls has been previously suggested in healthy adults aged $\geq 65$ years [24] and in diabetic patients with peripheral 

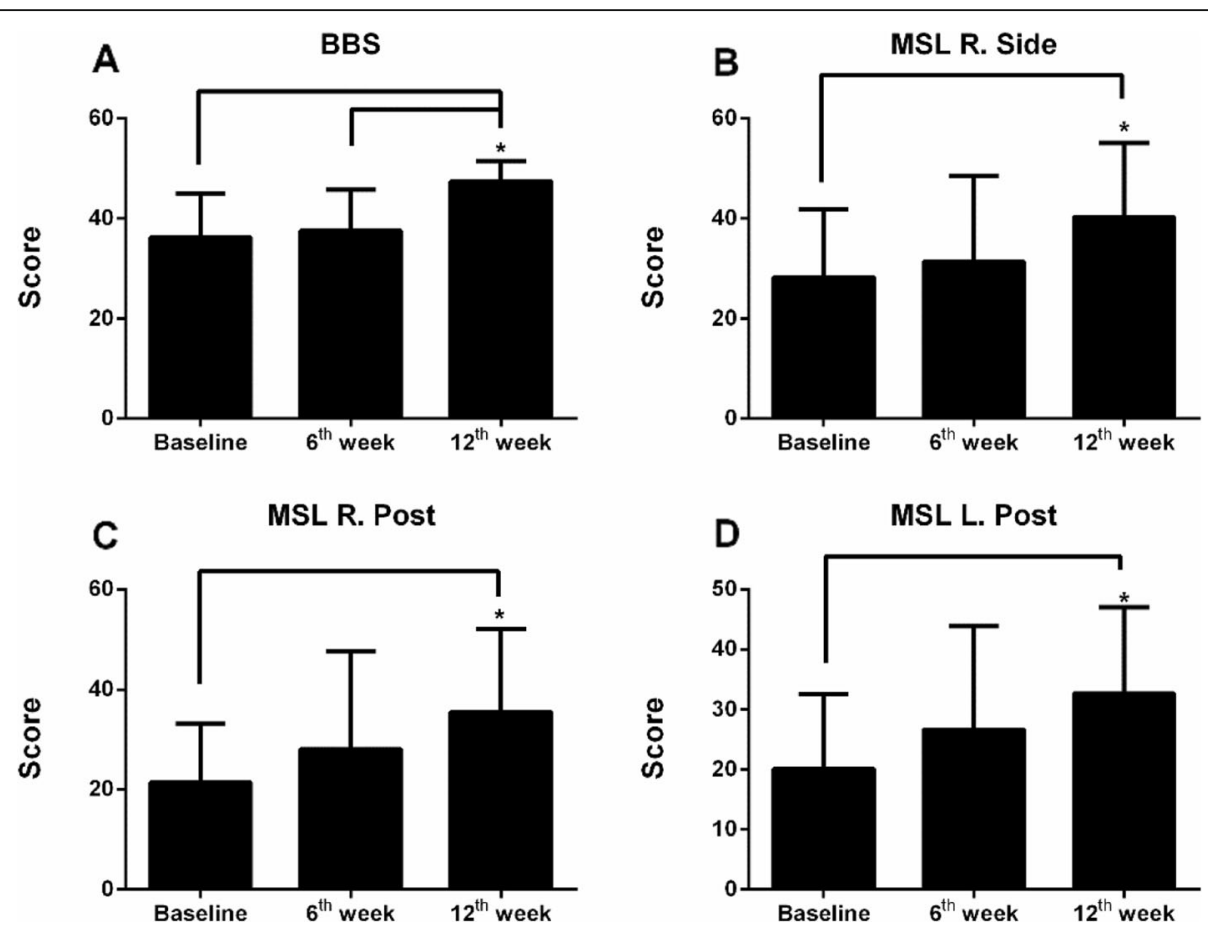

Fig. 4 Outcome measures collected at three time points in Group B. The scores were presented as mean \pm SD. Differences between time points were examined using repeated measures ANOVA, followed by a post hoc Bonferroni test. The alpha value was set to 0.05 for BBS and MFES; 0.0021 for subscales of SF-36; 0.0056 for subscales of MDRT; 0.0028 for subscales of MSL

neuropathy [23]. However, IVGB training did not significantly alter all subscales of SF-36 in the present study, indicating no obvious impact of IVGB training on quality of life in older patients with mild-to-moderate PD.

The stepping reaction, which is critical for successful recovery of balance, requires the integration of neural and sensorimotor control systems [39]. Previous studies indicated that stepping exercises on a treadmill and tango dancing increased balance and mobility in PD patients $[39,40]$. In addition, PD patients perform lateral weight shifting with less accuracy than do healthy controls [41]. The intervention using weight-shifting balance exercises significantly increases postural stability in the anterior-posterior and medial-lateral directions, which is commonly limited in PD patients [42]. It has been demonstrated that randomized rightward, leftward, and forward-backward perturbations improved protective postural responses in PD patients [43]. Consistently, we observed in the present study that multi-directional step task and target-directed stepping task of the IVGB exercise program improved motor coordination, the ability to stand on one leg, and functional stepping in older patients with mild-to-moderate PD, although not all subscales of MDRT and MSL were improved by IVGB training in all included PD patients.

The current customized IVGB exercise program may provide several benefits to older patients with mild-to- moderate PD. First, the included PD patients indicated that IVGB training with auditory and visual feedback more interesting, compared to conventional stepping and balance training. In addition, the real-time feedback regarding exercise performance during IVGB training gives PD patients a sense of achievement, thereby motivating them to do their best. Finally, the IVGB training program consists of the multiple direction stepping task and target-oriented stepping tasks of the IVGB training program address balance, postural control, and weightshifting ability; therefore, both tasks are suitable for motor rehabilitation in PD.

The benefits of virtual-reality-based physical exercises (exergames) in PD motor rehabilitation have been long demonstrated. Treadmill training with virtual obstacles improves physical performance and gait in older persons with PD [44]. Physical rehabilitation using a customized videogame ameliorates gait and balance problems in PD patients [45]. Furthermore, the Wii Fit Exercise substantially enhances obstacle-crossing performance and dynamic balance in patients with HY stage 1-3 PD [19], and similar promising results were reported in two RCTs $[17,20]$. On the other hand, the superiority of auditory cueing over visual cueing in PD rehabilitation has been suggested [13], and rhythmic auditory stimulation reinforced the beneficial effect of multidirectional step training on gait and balance in PD [46]. Rhythmic skills are 
Table 2 Between-group comparisons of the changes in outcome measures in the first and second 6-week periods

\begin{tabular}{|c|c|c|c|c|c|c|}
\hline & \multicolumn{3}{|c|}{ First 6-week period } & \multicolumn{3}{|c|}{ Second 6-week period } \\
\hline & Group A & Group B & $P$ value $^{a}$ & Group A & Group B & $P$ value $^{a}$ \\
\hline & \multicolumn{3}{|l|}{ Mean \pm SD } & \multicolumn{3}{|l|}{ Mean \pm SD } \\
\hline BBS (score) & $1.0 \pm 3.8$ & $1.4 \pm 6.7$ & 0.85 & $-3.1 \pm 4.9$ & $9.9 \pm 7.2$ & $<0.0001$ \\
\hline \multicolumn{7}{|l|}{ SF-36 (score) } \\
\hline Physical functioning & $9.6 \pm 10.5$ & $0 \pm 19.2$ & 0.14 & $2.5 \pm 13.6$ & $10.9 \pm 19.1$ & 0.23 \\
\hline Physical problems & $10.0 \pm 31.3$ & $-13.9 \pm 32.4$ & 0.09 & $-1.7 \pm 23.9$ & $18.8 \pm 28.5$ & 0.07 \\
\hline Emotional problems & $10.9 \pm 29.5$ & $-23.3 \pm 39.5$ & 0.02 & $-2.9 \pm 25.2$ & $29.6 \pm 35.2$ & 0.02 \\
\hline Vitality (energy/ fatigue) & $13.8 \pm 13.5$ & $2.9 \pm 25.1$ & 0.20 & $-0.4 \pm 21.4$ & $8.3 \pm 23.1$ & 0.35 \\
\hline General mental health & $11.4 \pm 21.5$ & $5.3 \pm 14.3$ & 0.42 & $-0.2 \pm 25.0$ & $5.8 \pm 22.5$ & 0.54 \\
\hline Social functioning & $14.2 \pm 34.2$ & $0.6 \pm 19.6$ & 0.25 & $0 \pm 0$ & $11.3 \pm 28.2$ & 0.19 \\
\hline Bodily pain & $5.6 \pm 31.6$ & $15.2 \pm 26.9$ & 0.43 & $1.7 \pm 35.5$ & $9.6 \pm 29.2$ & 0.56 \\
\hline General health & $6.7 \pm 34.7$ & $7.9 \pm 18.1$ & 0.91 & $4.6 \pm 35.3$ & $8.8 \pm 20.6$ & 0.73 \\
\hline MFES (score) & $8.3 \pm 17.0$ & $-8.1 \pm 14.1$ & 0.02 & $-6.5 \pm 15.0$ & $21.3 \pm 35.7$ & 0.03 \\
\hline \multicolumn{7}{|l|}{ MDRT (cm) } \\
\hline MDRT-F & $1.6 \pm 6.5$ & $3.1 \pm 6.5$ & 0.57 & $-1.7 \pm 6.2$ & $1.2 \pm 6.5$ & 0.29 \\
\hline MDRT-R & $8.5 \pm 7.4$ & $-0.5 \pm 5.5$ & 0.003 & $-2.7 \pm 8.6$ & $2.2 \pm 4.2$ & 0.09 \\
\hline MDRT-L & $6.9 \pm 5.9$ & $-0.8 \pm 5.8$ & 0.004 & $-2.7 \pm 8.3$ & $3.6 \pm 6.2$ & 0.05 \\
\hline \multicolumn{7}{|l|}{ MSL (cm) } \\
\hline R. Ant & $3.1 \pm 10.4$ & $4.9 \pm 12.4$ & 0.69 & $-2.3 \pm 8.2$ & $2.7 \pm 6.8$ & 0.12 \\
\hline L. Ant & $2.4 \pm 12.4$ & $2.8 \pm 4.4$ & 0.92 & $-1.2 \pm 9.7$ & $4.6 \pm 8.3$ & 0.13 \\
\hline R. Side & $2.6 \pm 8.7$ & $3.1 \pm 9.4$ & 0.89 & $-1.8 \pm 9.7$ & $8.9 \pm 10.4$ & 0.02 \\
\hline L. Side & $0.5 \pm 10.2$ & $-0.03 \pm 7.3$ & 0.89 & $-1.4 \pm 11.0$ & $7.5 \pm 9.1$ & 0.04 \\
\hline R. Post & $4.3 \pm 13.4$ & $6.7 \pm 8.9$ & 0.63 & $-6.4 \pm 9.3$ & $7.5 \pm 11.5$ & 0.004 \\
\hline L. Post & $2.9 \pm 14.3$ & $6.7 \pm 7.7$ & 0.43 & $-5.9 \pm 8.3$ & $5.9 \pm 9.2$ & 0.003 \\
\hline
\end{tabular}

SD standard deviation, BBS Berg Balance Scale, SF-36 the 36-Item Short-Form Health Survey, MFES Modified Falls Efficacy Scale, MDRT Multi-Directional Reach Test, $M D R T-F$ MDRT to the forward side, MDRT-R MDRT to the right side, MDRT-L MDRT to the left side, MSL Maximum Step Length, $R$. Ant right leg, anterior side, L. Ant left leg, anterior side, $R$. Side right side, $L$. Side left side, $R$. Post right leg, posterior side, $L$. Post left leg, posterior side

${ }^{a}$ The alpha value was set to 0.05 for BBS and MFES; 0.0031 for subscales of SF-36; 0.0083 for subscales of MDRT; 0.0042 for subscales of MSL. Significant difference after Bonferroni correction was indicated in bold

suggested to serve as a predictor of disease severity and recovery capacity upon auditory cueing in patients with HY stage 2 PD [47], and rhythm-based games (music games) enhance rhythmic skills and motor functions in PD patients [48]. Therefore, it is reasonable to assume that the integration of rhythmic stimulation (music) into the current IVGB exercise program may further strengthen its effectiveness in motor recovery in older patients with mild-to-moderate PD.

A recent study reported that a home-based, squarestepping exercise increased mobility and cognitive skills among older adults with multiple sclerosis [9]. Furthermore, a 6-week training using a music-based game at home improves rhythmic skills in patients with HY stage 2-3 PD [48]. In the present study, all IVGB training courses were held at a hospital in the presence of a physical therapist who was responsible for preventing falls. Further prospective studies with a larger sample size are warranted to evaluate the feasibility of home-based
IVGB training for PD patients; to determine the appropriate users in terms of age, disease severity, and comorbidities; and to develop relevant safety guidelines.

Although the physiological and neural mechanisms underlying the functional benefits of exergames remain to be investigated, the notion that exercise boosts synaptic strength and potentiates functional circuity in PD is widely accepted [49]. In addition, several possible neurobiological mechanisms have been proposed to underlie this relationship, including increased release of neurotransmitters, modulators, and tropic factors, as well as cognitive gains [50].

The present study had several limitations. First, the sample size was small. Second, the sex ratio and the mean MMSE score were unequal between the two groups, which might confound the results to some degree. Third, physiological variables such as heart rate and pulse rate were not assessed in this study. Finally, conventional motor rehabilitation was not included for 
comparison. Further investigation with a larger population of age-, sex-, mental status- and HY-stage-matched PD patients and longer follow-up periods is needed to confirm the effectiveness of IVGB training over that of conventional physiotherapy in PD rehabilitation.

\section{Conclusion}

A 6-week, hospital-based IVGB exercise program improved the balance, postural stability, and confidence in preventing falls in older patients with HY stage 1-3 PD. Thus, IVGB exercise training might serve as a rehabilitation regimen to ameliorate physical symptoms in older adults with mild-to-moderate PD.

\section{Supplementary information}

Supplementary information accompanies this paper at https://doi.org/10. 1186/s12984-020-00725-y.

Additional file 1: Supplemental Table 1. Outcome measures of two groups of PD patients at baseline, week 6 and week 12 .

\section{Consent for publication}

All participants provided signed informed consent for the publication of this study.

\section{Competing interests}

The authors declare no conflict of interests.

\section{Author details}

${ }^{1}$ Department of Neurology, School of Medicine, College of Medicine, Taipei Medical University, Taipei, Taiwan. ${ }^{2}$ Department of Neurology, Taipei Medical University Hospital, Taipei, Taiwan. ${ }^{3}$ Department of Physical Medicine and Rehabilitation, School of Medicine, College of Medicine, Taipei Medical University, Taipei, Taiwan. ${ }^{4}$ Department of Physical Medicine and Rehabilitation, Taipei Medical University Hospital, No. 252, Wu-Hsing St. Taipei City 110, Taiwan. ${ }^{5}$ Taipei Neuroscience Institute, Taipei Medical University, Taipei, Taiwan. ${ }^{6}$ School of Biomedical Engineering, College of Biomedical Engineering, Taipei Medical University, Taipei, Taiwan. ${ }^{7}$ Graduate Institute of Biomedical Optomechatronics, College of Biomedical Engineering, Taipei Medical University, Taipei, Taiwan. ${ }^{8}$ Graduate Institute of Injury Prevention and Control, Taipei Medical University, Taipei, Taiwan.

${ }^{9}$ Department of Physical Medicine and Rehabilitation, Wan Fang Hospital, Taipei Medical University, Taipei, Taiwan.

Received: 3 December 2019 Accepted: 6 July 2020

Published online: 13 July 2020

\section{References}

PD: Parkinson's disease; IVGB: Interactive video game-based; BBS: Berg Balance Scale; MFES: Modified Falls Efficacy Scale; UST: Unipedal Stance Test; TUG: Timed Up and Go; MMSE: Mini-Mental Status Examination; SF-36: 36Item Short-Form Health Survey; MDRT: Multi-Directional Reach Test; MSL: Maximum Step Length; Mean \pm SD: Mean and standard deviation; BMI: Body Mass Index; MDRT-F: Multi-Directional Reach Test to the front side; MDRT-R: Multi-Directional Reach Test to the right side; MDRT-L: MultiDirectional Reach Test to the left side; RCTs: Randomized controlled trials

\section{Acknowledgements}

We would like to thank the physical therapists Fan-Chien Chang and Sheng-Wen Su for monitoring the IVGB training courses. We also would like to thank Dr. YenKuang Lin from the Research Center of Biostatistics, Taipei Medical University, for statistical consultation and the team members of Supreme Investment Co. (Taipei, Taiwan) for providing the IVGB programs and technical support.

\section{Authors' contributions}

RYY confirmed the pathological diagnosis of participants, recruited participants, and interpreted data. SCC supplied the instruments and contributed to the study design. CWP collected the data and conducted data analysis. YNL analyzed and interpreted the data. YTC helped to recruit participants and corrected the data. $\mathrm{CHL}$ contributed substantially to the study design, drafted the manuscript, and revised the manuscript. All authors contributed to the writing of the manuscript and approved the final manuscript.

\section{Funding}

This work was supported by the Ministry of Science and Technology (grant number MOST-108-2221-E-038-008) and the Higher Education Sprout Project by the Ministry of Education (grant number DP2-109-21121-01-N-02-02) in Taiwan.

\section{Availability of data and materials}

The datasets generated during the current study are available from the corresponding author upon request.

\section{Ethics approval and consent to participate}

This study was conducted at Taipei Medical University Hospital, and the study protocol was reviewed and approved by the Joint Institutional Review Board of Taipei Medical University (approval number: TMU-JIRB 201311032). The potential risks and benefits of participation in this study were explained to each participant in advance. All participants provided signed informed consent before participation.
1. de Lau LM, Breteler MM. Epidemiology of Parkinson's disease. Lancet Neurol. 2006:5(6):525-35.

2. Dibble LE, Addison O, Papa E. The effects of exercise on balance in persons with Parkinson's disease: a systematic review across the disability spectrum. J Neurol Phys Ther. 2009;33(1):14-26.

3. Johansson C, et al. Balance and mobility in patients with newly diagnosed Parkinson's disease. Disabil Rehabil. 2020;42(6):770-8. https://doi.org/10. 1080/09638288.2018.1509240.

4. Crizzle AM, Newhouse IJ. Is physical exercise beneficial for persons with Parkinson's disease? Clin J Sport Med. 2006;16(5):422-5.

5. Rantakokko M, et al. Life-space mobility in Parkinson's disease: associations with motor and non-motor symptoms. J Gerontol A Biol Sci Med Sci. 2019; 74(4):507-12.

6. Goodwin VA, et al. The effectiveness of exercise interventions for people with Parkinson's disease: a systematic review and meta-analysis. Mov Disord. 2008;23(5):631-40

7. Lai $\mathrm{CH}$, et al. Exercise interventions for individuals with neurological disorders: a systematic review of systematic reviews. Am J Phys Med

8. Santos D, et al. Physical activity in older adults with mild Parkinsonian signs: a cohort study. J Gerontol A Biol Sci Med Sci. 2018;73(12):1682-7.

9. Sebastiao E, et al. Home-based, square-stepping exercise program among older adults with multiple sclerosis: results of a feasibility randomized controlled study. Contemp Clin Trials. 2018;73:136-44.

10. Dozza M, et al. What is the most effective type of audio-biofeedback for postural motor learning? Gait Posture. 2011;34(3):313-9.

11. Schlick $C$, et al. Visual cues combined with treadmill training to improve gait performance in Parkinson's disease: a pilot randomized controlled trial. Clin Rehabil. 2016;30(5):463-71.

12. Gallagher R, et al. Auditory and visual cueing modulate cycling speed of older adults and persons with Parkinson's disease in a virtual cycling (Vcycle) system. J Neuroeng Rehabil. 2016;13(1):77.

13. Spaulding SJ, et al. Cueing and gait improvement among people with Parkinson's disease: a meta-analysis. Arch Phys Med Rehabil. 2013;94(3):562-70.

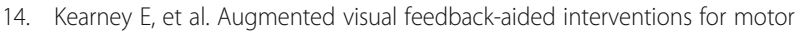
rehabilitation in Parkinson's disease: a systematic review. Disabil Rehabil. 2019;41(9):995-1011. https://doi.org/10.1080/09638288.2017.1419292.

15. Begel V, et al. Music games: potential application and considerations for rhythmic training. Front Hum Neurosci. 2017;11:273.

16. Barry G, Galna B, Rochester L. The role of exergaming in Parkinson's disease rehabilitation: a systematic review of the evidence. J Neuroeng Rehabil. 2014;11:33. Rehabil. 2019;98(10):921-30. 
17. Gandolfi M, et al. Virtual reality Telerehabilitation for postural instability in Parkinson's disease: a multicenter, single-blind, randomized. Controlled Trial Biomed Res Int. 2017;2017:7962826.

18. Garcia-Agundez A, et al. Recent advances in rehabilitation for Parkinson's disease with Exergames: a systematic review. J Neuroeng Rehabil. 2019; 16(1):17.

19. Liao $Y$ Y, et al. Virtual reality-based training to improve obstacle-crossing performance and dynamic balance in patients with Parkinson's disease. Neurorehabil Neural Repair. 2015;29(7):658-67.

20. Shih $M C$, et al. Effects of a balance-based exergaming intervention using the Kinect sensor on posture stability in individuals with Parkinson's disease: a single-blinded randomized controlled trial. J Neuroeng Rehabil. 2016;13(1):78

21. Zimmermann $\mathrm{R}$, et al. Cognitive training in Parkinson disease: cognitionspecific vs nonspecific computer training. Neurology. 2014;82(14):1219-26.

22. Betker $A L$, et al. Video game-based exercises for balance rehabilitation: a single-subject design. Arch Phys Med Rehabil. 2006;87(8):1141-9.

23. Hung ES-W, et al. Effects of interactive video game-based exercise on balance in diabetic patients with peripheral neuropathy: an open-level, Crossover Pilot Study. Evid Based Complement Altern Med. 2019;2019:9.

24. Lai $\mathrm{CH}$, et al. Effects of interactive video-game based system exercise on the balance of the elderly. Gait Posture. 2013;37(4):511-5.

25. Goetz CG, et al. Movement Disorder Society task force report on the Hoehn and Yahr staging scale: status and recommendations. Mov Disord. 2004; 19(9):1020-8.

26. Del Felice $\mathrm{A}$, et al. Personalized transcranial alternating current stimulation (tACS) and physical therapy to treat motor and cognitive symptoms in Parkinson's disease: a randomized cross-over trial. Neuroimage Clin. 2019;22: 101768.

27. Scalzo PL, et al. Validation of the Brazilian version of the berg balance scale for patients with Parkinson's disease. Arq Neuropsiquiatr. 2009;67(3b):831-5.

28. Pompeu JE, et al. Effect of Nintendo Wii-based motor and cognitive training on activities of daily living in patients with Parkinson's disease: a randomised clinical trial. Physiotherapy. 2012;98(3):196-204.

29. Ware JE Jr, Sherbourne CD. The MOS 36-item short-form health survey (SF-36). I. Conceptual framework and item selection. Med Care. 1992;30(6):473-83.

30. Morley D, et al. Predictors of activity and participation across neurodegenerative conditions: a comparison of people with motor neurone disease, multiple sclerosis and Parkinson's disease. BMC Neurol. 2018;18(1):19.

31. Steffen $T$, Seney M. Test-retest reliability and minimal detectable change on balance and ambulation tests, the 36-item short-form health survey, and the unified Parkinson disease rating scale in people with parkinsonism. Phys Ther. 2008;88(6):733-46.

32. Cherup NP, et al. Power vs strength training to improve muscular strength, power, balance and functional movement in individuals diagnosed with Parkinson's disease. Exp Gerontol. 2019;128:110740.

33. Newton RA. Validity of the multi-directional reach test: a practical measure for limits of stability in older adults. J Gerontol A Biol Sci Med Sci. 2001; 56(4):M248-52.

34. Cho BL, Scarpace D, Alexander NB. Tests of stepping as indicators of mobility, balance, and fall risk in balance-impaired older adults. J Am Geriatr Soc. 2004;52(7):1168-73.

35. Piccinini $G$, et al. The impact of cognitive reserve on the effectiveness of balance rehabilitation in Parkinson's disease. Eur J Phys Rehabil Med. 2018; 54(4):554-9.

36. Wen MC, et al. Depression, anxiety, and apathy in Parkinson's disease: insights from neuroimaging studies. Eur J Neurol. 2016;23(6):1001-19.

37. Pradhan S. The use of commercially available games for a combined physical and cognitive challenge during exercise for individuals with Parkinson's disease - a case series report. Physiother Theory Pract. 2019; 35(4):355-62.

38. van Santen J, et al. Effects of Exergaming in people with dementia: results of a systematic literature review. J Alzheimers Dis. 2018;63(2):741-60.

39. Hackney ME, McKee K. Community-based adapted tango dancing for individuals with Parkinson's disease and older adults. J Vis Exp. 2014;(94): e52066. https://doi.org/10.3791/52066.

40. Tseng IJ, Yuan RY, Jeng C. Treadmill training improves forward and backward gait in early Parkinson disease. Am J Phys Med Rehabil. 2015; 94(10):811-9.

41. van den Heuvel MRC, et al. The effects of visual feedback during a rhythmic weight-shifting task in patients with Parkinson's disease. Gait Posture. 2016; 48:140-5.
42. Fung A, Lai EC, Lee BC. Usability and validation of the smarter balance system: an unsupervised dynamic balance exercises system for individuals with Parkinson's disease. IEEE Trans Neural Syst Rehabil Eng. 2018;26(4):798-806.

43. Peterson DS, Dijkstra BW, Horak FB. Postural motor learning in people with Parkinson's disease. J Neurol. 2016;263(8):1518-29.

44. Mirelman A, et al. Virtual reality for gait training: can it induce motor learning to enhance complex walking and reduce fall risk in patients with Parkinson's disease? J Gerontol A Biol Sci Med Sci. 2011;66(2):234-40.

45. Nuic $D$, et al. The feasibility and positive effects of a customised videogame rehabilitation programme for freezing of gait and falls in Parkinson's disease patients: a pilot study. J Neuroeng Rehabil. 2018;15(1):31.

46. Kadivar $Z$, et al. Effect of step training and rhythmic auditory stimulation on functional performance in Parkinson patients. Neurorehabil Neural Repair. 2011;25(7):626-35

47. Cochen De Cock V, et al. Rhythmic abilities and musical training in Parkinson's disease: do they help? NPJ Parkinsons Dis. 2018;4:8.

48. Dauvergne $C$, et al. Home-based training of rhythmic skills with a serious game in Parkinson's disease: usability and acceptability. Ann Phys Rehabil Med. 2018;61(6):380-5.

49. Abbruzzese $\mathrm{G}$, et al. Rehabilitation for Parkinson's disease: current outlook and future challenges. Parkinsonism Relat Disord. 2016;22(Suppl 1):S60-4.

50. Costa MTS, et al. Virtual reality-based exercise with Exergames as medicine in different contexts: a short review. Clin Pract Epidemiol Ment Health. 2019; 15:15-20.

\section{Publisher's Note}

Springer Nature remains neutral with regard to jurisdictional claims in published maps and institutional affiliations.

Ready to submit your research? Choose BMC and benefit from:

- fast, convenient online submission

- thorough peer review by experienced researchers in your field

- rapid publication on acceptance

- support for research data, including large and complex data types

- gold Open Access which fosters wider collaboration and increased citations

- maximum visibility for your research: over $100 \mathrm{M}$ website views per year

At BMC, research is always in progress.

Learn more biomedcentral.com/submissions 